\title{
Three Design Aspects of Multicarrier Interleave Division Multiple Access
}

\author{
Rong Zhang and Lajos Hanzo, Fellow, IEEE
}

\begin{abstract}
This paper presents a novel generalized transceiver architecture, i.e., Multicarrier Interleave-Division-Multiplexingaided Interleave Division Multiple Access (MC-IDM-IDMA). A parallel interference cancellation (PIC)-aided iterative multiuser detection (MUD) algorithm is introduced, and the convergence behavior of the system is evaluated with the aid of EXtrinsic Information Transfer (EXIT) charts under the assumption of an equal-power multiuser scenario. Three interesting design tradeoffs of the MC-IDM-IDMA system are analyzed: 1) the multiplexing versus diversity tradeoffs; 2) the coding versus spreading tradeoffs, where the achievable bandwidth efficiency of both MC-IDM and MC-IDMA are characterized, and a general design rule is provided for low-rate codes; and 3) the complexity versus performance tradeoffs and the EXIT characteristics of two different MUD algorithms are investigated, leading to a reducedcomplexity hybrid MUD.
\end{abstract}

Index Terms-EXtrinsic Information Transfer (EXIT) charts, interleave division multiplexing/interleave division multiple access (IDM/IDMA), multicarrier (MC), multiuser detection (MUD).

\section{INTRODUCTION}

B ASED ON the chip-interleaved code-division multipleaccess (CDMA) philosophy of Frenger et al. [1] and Mahadevappa and Proakis [2], the interleave-division multipleaccess (IDMA) philosophy was further developed by Ping et al. [3], [4] and Schoeneich and Hoeher [5], [6]. IDMA entails reversing the classic position of direct sequence (DS) spreading $\left(S_{k}\right)$ and interleaving $(\pi)$ employed in traditional CDMA systems [7], leading to chip interleaving instead of bit interleaving, where the different users are distinguished by their unique userspecific chip interleavers $\pi_{k}$. The benefits of single-carrier (SC) IDMA are the following: 1) random coding, ${ }^{1}$ where different users have unique random signatures; 2) time diversity, where chip interleaving is capable of increasing the achievable time diversity in time-selective channels; and 3) joint coding and spreading design, which is capable of improving the attainable bandwidth efficiency and error resilience.

Manuscript received June 12, 2007; revised November 2, 2007, January 22, 2008, and January 28, 2008. First published February 15, 2008; current version published November 12, 2008. This work was supported by and formed part of the Core 4 Research Program of the Virtual Centre of Excellence in Mobile and Personal Communications, Mobile VCE (www.mobilevee.com), and was also supported by EPSRC. The review of this paper was coordinated by Prof. J. Wu.

The authors are with the School of Electronics and Computer Science, University of Southampton, SO17 1BJ Southampton, U.K.

Digital Object Identifier 10.1109/TVT.2008.918724

\footnotetext{
${ }^{1} \mathrm{~A}$ code is said to be random if its elements are independent and identically distributed (i.i.d), which is optimal when aiming for maximizing the mutual information from an information-theoretical point of view.
}

The discussions of SC-IDMA found in the open literature were concentrated on three aspects, namely, low-rate code design [8], [9], power allocation [4], and practical implementation [10], [11]. Low-rate turbo-Hadamard codes [8] and zig-zag codes [9] were employed in IDMA designed for time-hoppingaided ultrawideband (UWB) systems by Ping et al. [12]. The joint code rate and spreading factor (SF) design of SC-IDMA was addressed in [13], whereas the power allocation was optimized in [4], where a linear-programming-based power allocation was used. The practical implementation of the channel estimation was addressed in [10]. Furthermore, asynchronous uplink (UL) transmission and the achievable near-far resistance of SC-IDMA were compared to that of DS-CDMA in [11], where a similar performance was observed for both. Owing to its meritorious properties, IDMA has been proposed for numerous applications, such as next-generation cellular UL systems [5], [14] and UWB systems [12], as well as for ad hoc networks [15]. Various information-theoretic aspects of IDMA were discussed in [6] and [16].

Recently, the multicarrier (MC) version of IDMA was proposed in [17] and [18], but at the time of this writing, no journal paper addresses the rigorous design of the MC-IDMA scheme, which may be viewed as an MC-CDMA system having both time-domain (TD) and frequency-domain (FD) chip interleaving or frequency hopping. Thus, MC-IDMA is more resilient to correlated subcarrier fading experienced in low-dispersion channels. Furthermore, since spreading is used before interleaving, the resultant chips are randomly mapped to different time and frequency slots, resulting in both TD and FD spreading. Thus, MC-IDMA benefits from both TD and FD diversity.

In this paper, we generalize UL MC-IDMA, leading to the concept of UL MC Interleave-Division-Multiplexing-aided IDMA (IDM-IDMA), where each user of the original MCIDMA system [18] transmits multiple streams differentiated by stream-specific chip interleavers. This concept is similar to the multicode transmission regime employed in the highspeed packet access (HSPA) system [19]. As a special case of our general concept, MC-IDM can be considered as a downlink (DL) broadcast system scenario, whereas MC-IDMA may be considered as a UL multiple-access system. An attractive feature of the generalized MC-IDM-IDMA system is that it employs an iterative receiver [20], [21], which strikes an attractive tradeoff between the potentially high complexity of optimum joint detection and decoding, as well as the potential performance loss imposed by separate detection and singleuser decoding. We analyze the system with the aid of EXtrinsic 
Information Transfer (EXIT) charts [22], [23] and investigate three interesting design aspects of the MC-IDM-IDMA system, namely, the associated multiplexing versus diversity tradeoffs, the coding versus spreading tradeoffs, and the complexity versus performance tradeoffs.

The novelty and rationale of this paper can be summarized as follows.

1) Multiplexing versus diversity tradeoffs. We compare the difference between an MC-IDM system and an MCIDMA system, explicitly quantifying the multiplexing gain versus diversity gain tradeoffs in our proposed MCIDM-IDMA system.

2) Coding versus spreading tradeoffs. The bandwidth efficiency of both MC-IDM and MC-IDMA systems can be improved by jointly designing the channel-coding rate and the SF. Hence, we will discuss the coding versus spreading tradeoffs in conjunction with various channel codes and provide design guidelines for choosing a lowrate code for both systems. The joint code rate and SF design was discussed in the context of CDMA systems in [24] and [25] and the references therein. It was found that the conclusions of joint code rate and SF design depend not only on the choice of specific forward errorcorrection (FEC) codes but on the properties of the multiuser detection (MUD) algorithms employed [26] as well. By contrast, in both MC-IDM and MC-IDMA systems, the MUD is carried out on a chip-by-chip basis, which implies that the joint code rate and SF design is equivalent to the design of an amalgamated low-rate channel-coding scheme.

3) Complexity versus performance tradeoffs. To enhance the achievable system load, we investigate two different MUDs using EXIT charts, namely, the optimum maximum-likelihood (ML) MUD and the suboptimum parallel interference cancellation (PIC) scheme. Their different characteristics are discussed, and a reducedcomplexity hybrid MUD concept is proposed. As an alternative approach, efforts were also dedicated to improving the system load supported with the aid of optimum power allocation and successive interference cancellation (SIC) during the MUD iterations.

This paper is organized as follows. In Section II, a brief system overview is presented, whereas in Section III, the EXIT chart analysis of MC-IDMA is provided. In Section IV, we discuss the aforementioned three tradeoffs involved in the design of our MC-IDM-IDMA system, namely, the multiplexing versus diversity tradeoffs, the coding versus spreading tradeoffs, and the complexity versus performance tradeoffs. Finally, we conclude in Section V.

\section{SYSTEM OVERVIEW}

\section{A. Bandwidth Expansion}

IDMA does not necessarily constitute a spread-spectrum system. However, to support multiple users in the DS spreading code domain, typically, DS spreading is also actively employed

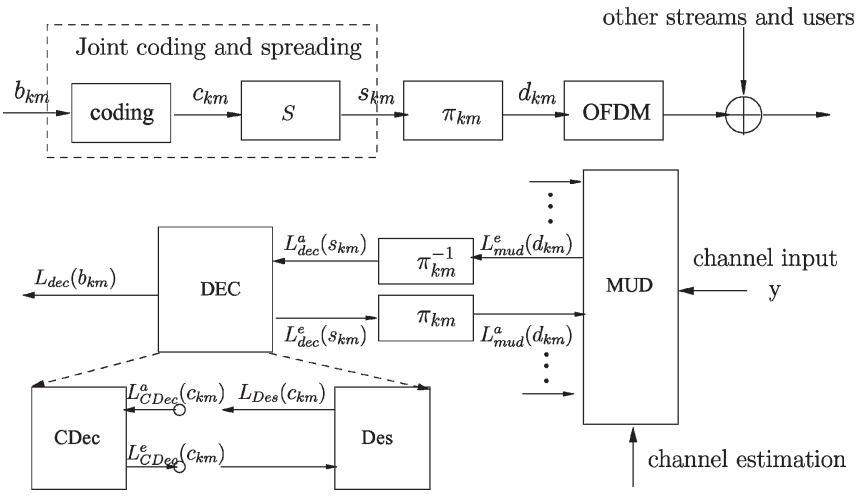

Fig. 1. Block diagram of the MC-IDM-IDMA transceiver corresponding to the $k$ th user's $m$ th stream.

since its employment provides an additional degree of design freedom [27]. An important advantage of IDMA is that it facilitates the flexible joint optimization of the code rate and SF using, for example, low-rate codes or diverse serial concatenated codes (SCC) [28]. A specific example is constituted by the concatenation of high-rate FEC codes and low-complexity repetition codes constituted by DS spreading, since spreading can be viewed as repetition coding followed by user-specific scrambling or interleaving [1].

In an SCC-aided IDMA system, given a fixed total bandwidth expansion factor of $\Omega$, we may jointly quantify the bandwidth expansion imposed by FEC coding having a code rate of $R$ and that by DS spreading having an SF of $G$, yielding $\Omega=G / R$. For the sake of characterizing the achievable throughput of the IDMA system, we define the normalized user load as

$$
\eta=\frac{K_{\max }}{\Omega}
$$

where $K_{\max }$ is the maximum number of users supported given a fixed bandwidth expansion factor $\Omega$ and $E_{b} / N_{0}$ value. Note that for a fixed bandwidth expansion factor $\Omega$ and a channel code rate $R$, the affordable SF is determined. Hence, $K_{\max }$ is a function of $\Omega, R$, and $E_{b} / N_{0}$. In case of an IDM system, we may accordingly define the normalized per-user throughput as $\eta=M_{\max } / \Omega$, where $M_{\max }$ is the maximum number of bit streams supported.

\section{B. Transceiver Architecture}

We consider a general MC-IDM-IDMA UL system where each of the $K$ users has $M_{k}$ channel-coded and DS spread multiplexed chip streams distinguished by stream-specific chip interleavers $\pi_{k m}$, as shown in Fig. 1. The system employs BPSK modulation for communicating over $K$ independent dispersive Rayleigh fading channels. Following orthogonal frequency-division multiplexing (OFDM) [29], each subcarrier experiences flat fading, and when a sufficiently long random chip interleaver is employed in both TD and FD, the corresponding FD channel transfer function (FDCTF) of each subcarrier may be assumed to be uncorrelated. We use the simplifying assumption of perfect FDCTF knowledge at the 
receiver employing a single antenna. The received signal can be expressed as

$$
y[n, i]=\sum_{k=1}^{K} H_{k}[n, i] \sum_{m=1}^{M_{k}} d_{k m}[n, i]+\nu[n, i]
$$

where $d_{k m}[n, i]$ denotes the $k$ th user's $m$ th stream's $i$ th subcarrier in the $n$th OFDM symbol, $H_{k}[n, i]$ is the FDCTF corresponding to user $k$ at the $i$ th subcarrier of the $n$th OFDM symbol, and $\nu$ is the additive white Gaussian noise (AWGN) having zero mean and a variance of $2 \sigma_{n}^{2}$. It is noted that our generalized system model represents a DL MC-IDM system when we have $K=1$, where all streams experience the same FDCTF. By contrast, a UL MC-IDMA system is modeled, where we have $M_{k}=1 \forall k$. Furthermore, we assume that each user has the same number of streams, i.e., $M_{k}=M \forall k$.

The receiver of MC-IDM-IDMA consists of a soft-in-softout (SISO) MUD and a bank of $K M$ individual SISO decoders (DEC), which are constituted by the combined channel decoder (CDec) and Despreader (Des), as shown in Fig. 1. The soft information exchanged between the receiver components is constituted by the extrinsic log-likelihood ratios (LLRs) [28]. The SISO MUD employs a matched filter (MF)-based soft PIC on a chip-by-chip basis [3].

We now consider the $i$ th subcarrier of the $n$th OFDM symbol. Hereafter, we omit the index $i$ and $n$ for notational simplicity. At the $l$ th iteration, the MUD outputs its extrinsic information $L_{\text {mud }}^{e}\left(d_{k m}\right)$ based on the channel input $y$ combined with the a priori information $L_{\mathrm{dec}}^{e}\left(d_{k m}\right)$ provided by the DEC. When considering the $k$ th user's $m$ th stream, we have

$$
y=H_{k} d_{k m}+\xi
$$

where $\xi=\sum_{j \neq k} H_{j} \sum_{m=1}^{M} d_{j m}+H_{k} \sum_{p \neq m} d_{k p}+\nu$ represents the interference plus noise. Let $I m$ and $R e$ represent the imaginary and real parts of a complex number, respectively. In case of BPSK modulation, the real part of $H_{k}^{*} y$ constitutes sufficient statistics for estimating $d_{k m}$, where $(\cdot)^{*}$ denotes conjugation, resulting in

$$
\operatorname{Re}\left(H_{k}^{*} y\right)=\left|H_{k}\right|^{2} d_{k m}+\operatorname{Re}\left(H_{k}^{*} \xi\right) .
$$

We denote the soft estimate of a variable $a$ by $(\hat{a})$. Then, $\operatorname{Re}\left(H_{k}^{*} \hat{\xi}\right)$ and its instantaneous power $V\left[\operatorname{Re}\left(H_{k}^{*} \hat{\xi}\right)\right]$ are given by

$$
\begin{aligned}
\operatorname{Re}\left(H_{k}^{*} \hat{\xi}\right)= & H_{k}^{R e} \hat{y}^{R e}+H_{k}^{I m} \hat{y}^{I m}-\left|H_{k}\right|^{2} \hat{d}_{k m} \\
V\left[R e\left(H_{k}^{*} \hat{\xi}\right)\right]= & \left(H_{k}^{R e}\right)^{2} V\left(\hat{y}^{R e}\right)+\left(H_{k}^{I m}\right)^{2} V\left(\hat{y}^{I m}\right) \\
& -\left|H_{k}\right|^{4} V\left(\hat{d}_{k m}\right)+2 H_{k}^{R e} H_{k}^{I m} \phi
\end{aligned}
$$

where $\phi=\sum_{k=1}^{K} H_{k}^{R e} H_{k}^{I m} \sum_{m=1}^{M} V\left(\hat{d}_{k m}\right)$, and the soft estimate $\hat{y}$ and its instantaneous power are thus expressed as

$$
\hat{y}^{R e}=\sum_{k=1}^{K} H_{k}^{R e} \sum_{m=1}^{M} \hat{d}_{k m}
$$

$$
\begin{aligned}
\hat{y}^{I m} & =\sum_{k=1}^{K} H_{k}^{I m} \sum_{m=1}^{M} \hat{d}_{k m} \\
V\left(\hat{y}^{R e}\right) & =\sum_{k=1}^{K}\left(H_{k}^{R e}\right)^{2} \sum_{m=1}^{M} V\left(\hat{d}_{k m}\right)+\sigma_{n}^{2} \\
V\left(\hat{y}^{I m}\right) & =\sum_{k=1}^{K}\left(H_{k}^{I m}\right)^{2} \sum_{m=1}^{M} V\left(\hat{d}_{k m}\right)+\sigma_{n}^{2} .
\end{aligned}
$$

The soft estimate $\hat{d}_{k m}$ can be represented as $\hat{d}_{k m}=$ $\tanh \left(L_{\mathrm{dec}}^{e}\left(d_{k m}\right) / 2\right)$, whereas its instantaneous power is given by $V\left(\hat{d}_{k m}\right)=1-\hat{d}_{k m}^{2}$. Assuming $\xi$ is Gaussian distributed, the extrinsic information $L_{\text {mud }}^{e}\left(d_{k m}\right)$ is given by

$$
L_{\text {mud }}^{e}\left(d_{k m}\right)=2\left|H_{k}\right|^{2} \frac{\operatorname{Re}\left(H_{k}^{*} y\right)-\operatorname{Re}\left(H_{k}^{*} \hat{\xi}\right)}{V\left[\operatorname{Re}\left(H_{k}^{*} \hat{\xi}\right)\right]} .
$$

Then, the extrinsic information gleaned from the MUD is forwarded as a priori information to the DEC, which computes a more reliable extrinsic information $L_{\mathrm{dec}}^{e}\left(d_{k m}\right)$ for the next iteration. The iterations are terminated when a predefined termination criterion is satisfied. Finally, the LLR $L_{\mathrm{dec}}\left(b_{k m}\right)$ of Fig. 1, which represents the original information bits, is subjected to a soft/hard decision.

\section{EXIT ChART ANALYSIS OF MC-IDMA}

In this section, before carrying out the PIC-aided MC-IDMA iterative receiver's EXIT-chart analysis, we first introduce the EXIT functions of a general iterative receiver and describe the two component EXIT functions of the IDMA system.

\section{A. EXIT Functions for Iterative Receiver}

EXIT charts were introduced by ten Brink [22] and were used to analyze iterative receivers designed for CDMA systems by Wang [23]. This technique relies on computing the mutual information of two constituent components, namely, that of the SISO MUD and SISO DEC, which are denoted by $I_{\text {mud }}^{a}$, $I_{\mathrm{mud}}^{e}, I_{\mathrm{dec}}^{a}$, and $I_{\mathrm{dec}}^{e}$. The accuracy of this technique relies on the assumption of using a sufficiently high transmission block length and an infinite-duration random chip interleaver for supporting a large number of users $K$ or streams $M$, when the resultant extrinsic information provided by the SISO MUD for the $k$ th SISO DEC $L_{\text {mud, } k}^{e}$ converges to the Gaussian distribution described as [30]

$$
L_{\text {mud }, k}^{e} \sim \mathcal{N}\left(2 \rho_{\text {mud }, k}^{e}, 4 \rho_{\text {mud }, k}^{e}\right)
$$

where $\rho_{\text {mud }, k}^{e}$ is the asymptotic output signal-to-noise ratio (SNR) of the SISO MUD. The available extrinsic information $L_{\text {mud, } k}^{e}$, which may be of limited benefit in highly interferencelimited scenarios, acts as the input of the SISO DEC in Fig. 1. The extrinsic information $L_{\mathrm{dec}, k}^{e}$ gleaned from the SISO DEC 
can also be approximated by a Gaussian distribution formulated as [30]

$$
L_{\mathrm{dec}, k}^{e} \sim \mathcal{N}\left(2 \rho_{\mathrm{dec}, k}^{e}, 4 \rho_{\mathrm{dec}, k}^{e}\right)
$$

where $\rho_{\mathrm{dec}, k}^{e}$ is the asymptotic output SNR of the SISO DEC. The corresponding mutual information $I(L ; X)$ in terms of $L_{\mathrm{mud}, k}^{e}$ and $L_{\mathrm{dec}, k}^{e}$ is calculated as

$$
I=\frac{1}{2} \sum_{x \in( \pm 1)} \int_{-\infty}^{+\infty} p(l \mid x) \log _{2} \frac{2 p(l \mid x)}{p(l \mid+1)+p(l \mid-1)} d l
$$

where $I(L ; X) \in[0,1]$, and $p(l \mid x)$ is the distribution of the extrinsic LLRs. The EXIT function of the SISO MUD is denoted by $T_{\text {mud }}$, whereas that of the SISO DEC by $T_{\mathrm{dec}}$. The flow of mutual information can be written as

$$
\begin{aligned}
I_{\text {mud }, k}^{e, l} & =T_{\text {mud }}\left(I_{\text {mud }, j}^{a, l} \quad \forall j \neq k\right) \\
& =T_{\text {mud }}\left(I_{\text {dec }, j}^{e, l-1} \quad \forall j \neq k\right) \\
& =T_{\text {mud }}\left(T_{\text {dec }}\left(I_{\text {dec }, j}^{a, l-1}\right) \quad \forall j \neq k\right) \\
& =T_{\text {mud }}\left(T_{\text {dec }}\left(I_{\text {mud }, j}^{e, l-1}\right) \quad \forall j \neq k\right)
\end{aligned}
$$

where $l$ denotes the iteration index, whereas $j$ and $k$ denote the user index. It is clear from (16) that the mutual information $I_{\text {mud }, k}^{e, l}$ of user $k$ depends on its previous value $I_{\text {mud, }, j}^{e, l-1}$ encountered for all other users, as suggested by the nonlinear functions $T_{\mathrm{dec}}$ and $T_{\mathrm{mud}}$. The mutual information $I_{\mathrm{mud}, k}^{e, l}$ is a function of $\rho_{\text {mud, } k}^{e, l}$ according to (13), which obeys

$$
\rho_{\text {mud }, k}^{e, l}=\frac{P_{k}}{\sigma^{2}+P_{I}^{l}}
$$

where $P_{k}$ and $P_{I}^{l}$ denote the signal power of the desired user $k$ and the residual interference power at iteration $l$, respectively. It is worth noting that in the current system context, all the individual users' signals affect the shape of the EXIT chart, and hence, a $(K+1)$-dimensional EXIT chart would be necessary if the $K$ users had different received signal powers. However, provided that all the users' signal is received at the same power, their mutual information may be replaced by the corresponding average, which allows us to use a conventional 2-D EXIT chart.

Thus, the employment of 2-D EXIT charts is appropriate when we study PIC and in the context of a system communicating over an AWGN channel or over a fading channel subjected to uncorrelated fading in either FD or TD, provided that all the users' signals are received at equal power, i.e., when accurate power control is possible.

\section{B. Investigation of $T_{\mathrm{mud}}$}

Let us now investigate the EXIT chart of the iterative IDMA receiver for transmission over an AWGN channel. First of all, let us investigate the MUD's EXIT function $T_{\text {mud }}$ shown in Fig. 2. The curves converge to points $\mathrm{A}, \mathrm{B}$, and $\mathrm{C}$ at the

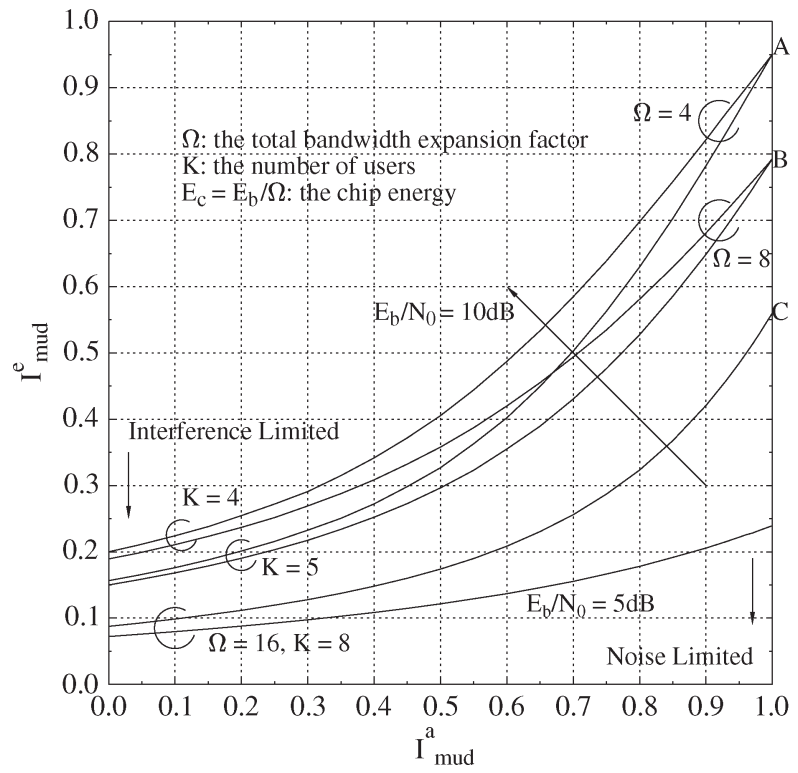

Fig. 2. MUD's EXIT charts.

operational point of $E_{b} / N_{0}=10 \mathrm{~dB}$, where $E_{b}$ is the bit energy, and $N_{0}$ is the power spectral density of the noise. Let us denote the number of users by $K$ and the bandwidth expansion factor by $\Omega$, yielding a chip energy of $E_{c}=E_{b} / \Omega$. We can then draw the following conclusions from Fig. 2.

1) Let us first investigate the left-hand-side area of Fig. 2, where $I_{\text {mud }}^{a}$ is low. In this area, we have an unreliable estimate of the multiuser interference (MUI), and hence, the residual interference power after PIC remains high, resulting in a low signal-to-interference-plus-noise ratio (SINR) or, equivalently, in low extrinsic mutual information $I_{\text {mud }}^{e}$, which is highly interference limited. This can be verified by the curves corresponding to $K=5$, which represent a lower $I_{\text {mud }}^{e}$ value than the curves recorded for $K=4$ users, as shown in Fig. 2.

2) Let us now consider the right-hand-side area of Fig. 2, where $I_{\text {mud }}^{a}$ is high. In this area, we benefit from a reliable estimation of the MUI since the interference was essentially cancelled, resulting in a high SINR or, equivalently, in a high extrinsic mutual information $I_{\text {mud }}^{e}$. Consequently, this scenario may be viewed as noise lim$i t e d$. Let us now consider the two curves having $E_{b} / N_{0}=$ $10 \mathrm{~dB}$ and using $\Omega=4$ (or $\Omega=8$ ). Although they correspond to different numbers of users, namely, $K=4$ and $K=5$, they both converge to the same point upon increasing $I_{\text {mud }}^{a}$.

3) Since the extrinsic mutual information $I_{\text {mud }}^{e}$ defined in (16) is a monotonically increasing function of $\rho_{\text {mud }, k}^{e}$ in (13) of the corresponding Gaussian-distributed LLRs [30], increasing the MUI or reducing the operating $E_{b} / N_{0}$ value results in a reduced $\rho_{\text {mud, } k}^{e}$ and, hence, in a reduced extrinsic mutual information $I_{\text {mud }}^{e}$ over the whole range of the a priori mutual information $I_{\mathrm{mud}}^{a}$. Hence, the shape of $T_{\text {mud }}$ is maintained.

4) A final interesting point may be observed by comparing the three extrinsic mutual information points $A, B$, and $C$ in Fig. 2, corresponding to $\Omega=4, \Omega=8$, and $\Omega=16$ 


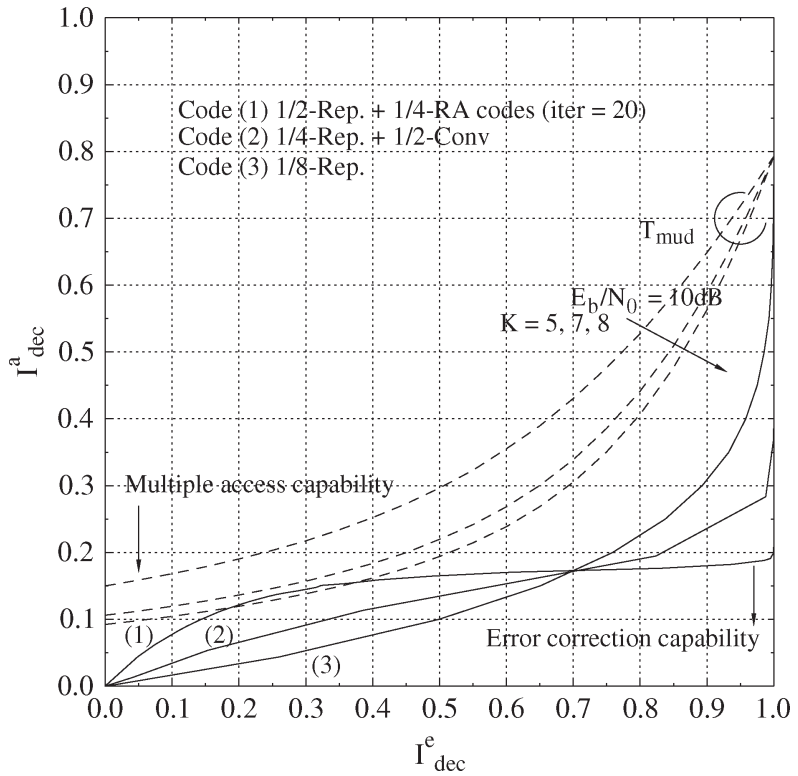

Fig. 3. EXIT charts of the various CDecs.

in conjunction with the perfect a priori mutual information of $I_{\text {mud }}^{a}=1$ at $E_{b} / N_{0}=10 \mathrm{~dB}$. Since the extrinsic mutual information is measured on a chip-by-chip basis, given the same $E_{b} / N_{0}$ value, the larger the bandwidth expansion factor, the lower the output $E_{c} / N_{0}$ value.

\section{Investigation of $T_{\mathrm{dec}}$}

We now investigate the EXIT curve of $T_{\text {dec }}$ shown in Fig. 3, where three different codes are considered in conjunction with a total bandwidth expansion factor of $\Omega=8$. More specifically, we consider three combinations: 1) Code 1, i.e., the concatenation of a rate-1/4 Repeat Accumulate (RA) [31] $\operatorname{code}^{2}$ and a rate- $1 / 2$ repetition code; 2 ) Code 2 , i.e., the concatenation of a rate- $1 / 2$ convolutional code and a rate- $1 / 4$ repetition code; and finally, 3) Code 3 , i.e., a standalone rate- $1 / 8$ repetition code. The MUD's EXIT curves are also shown in Fig. 3, corresponding to $K=5,7,8$ at $E_{b} / N_{0}=10 \mathrm{~dB}$. Based on Fig. 3, we arrive at the following conclusions.

1) As discussed before, the left-hand-side area of Fig. 3 represents an interference-limited region. Hence, the higher the number of users, the lower the extrinsic mutual information $I_{\text {mud }}^{e}$. Upon increasing the number of users and, hence, the MUI, the MUD's EXIT curve would first touch Code 1 in Fig. 3, then Code 2, and, finally, Code 3, indicating that the latter scenario of Code 3 is capable of supporting the highest number of users at a given SNR. Hence, we refer to this area of the EXIT chart as the region of high multiple-access capability.

\footnotetext{
${ }^{2}$ We consider a nonsystematic regular RA code, which may be viewed as a serial concatenated code having components separated by interleavers, where the outer code is a simple repetition code having a repetition length of $L$, whereas the inner code is a Unity-rate Recursive Convolutional (URC) code, which is also often referred to as an accumulator. The interleaver $\pi$ plays an essential role in RA codes, which can also be interpreted as a generator matrix. Hence, RA codes may also be considered as a class of low-density parity-check (LDPC) codes. The sum-product algorithm is used for decoding.
}

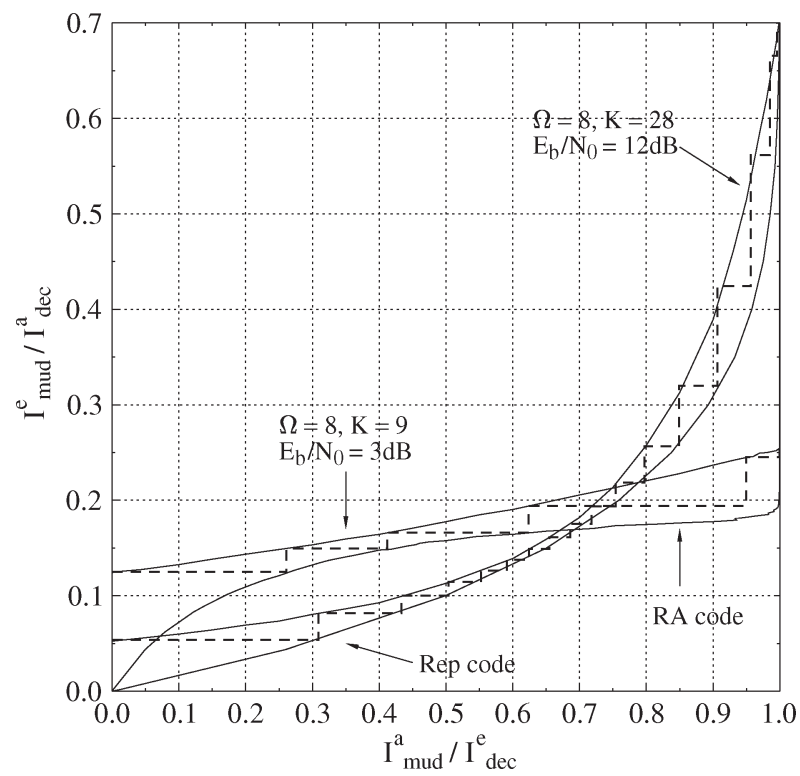

Fig. 4. Maximum number of users supported by the 128-subcarrier MC-IDMA system in a five-path chip-spaced negative exponentially decaying uncorrelated Rayleigh fading channel.

2) By contrast, the right-hand-side area of Fig. 3 is again referred to as being noise limited, where the MUI was essentially cancelled. As expected, the near-capacity RA code achieves $I_{\mathrm{dec}}^{e}=1$ for the lowest a priori mutual information of $I_{\mathrm{dec}}^{a}=0.2$, as shown in Fig. 3, which implies a low $E_{b} / N_{0}$ value. Hence, we refer to this area as a region of high error correction capability.

\section{EXIT Chart of MC-IDMA}

Let us now consider the EXIT chart analysis of MC-IDMA having 128 subcarriers, where the CIR encountered in this paper was an independently generated five-path chip-spaced negative exponentially decaying dispersive channel. Each path experienced uncorrelated Rayleigh fading. The difference in comparison to the previously considered AWGN channel manifests itself in terms of a different EXIT function $T_{\text {mud }}$, which takes into account the CIR knowledge. The resultant EXIT curve $T_{\mathrm{dec}}$ of the outer decoder can be modeled as in the previously considered AWGN scenario, since the outer channel DEC is fed with the near-Gaussian distributed output extrinsic information of the MUD. Let us now consider the accuracy of our EXIT chart analysis in a MC-IDMA scenario. Observe in Fig. 4 that a maximum of $K=28$ users were supported by Code 3 at $E_{b} / N_{0}=12 \mathrm{~dB}$. By contrast, $K=9$ users were serviced by Code 1 at $E_{b} / N_{0}=3 \mathrm{~dB}$, where the maximum number of users was found by identifying the EXIT curves of the MUD and DEC, where they "just" touch each other. The corresponding simulation trajectory in Fig. 4 confirms the convergence and accuracy of our EXIT chart analysis. Furthermore, the corresponding bit error rate (BER) simulation results were portrayed in Fig. 5, whereas as many as $K=28$ users were supported by Code 3 at $E_{b} / N_{0}=12 \mathrm{~dB}$. By contrast, Code 1 supported $K=9$ users at $E_{b} / N_{0}=3 \mathrm{~dB}$ and $K=16$ users at $E_{b} / N_{0}=12 \mathrm{~dB}$, confirming a good agreement with 


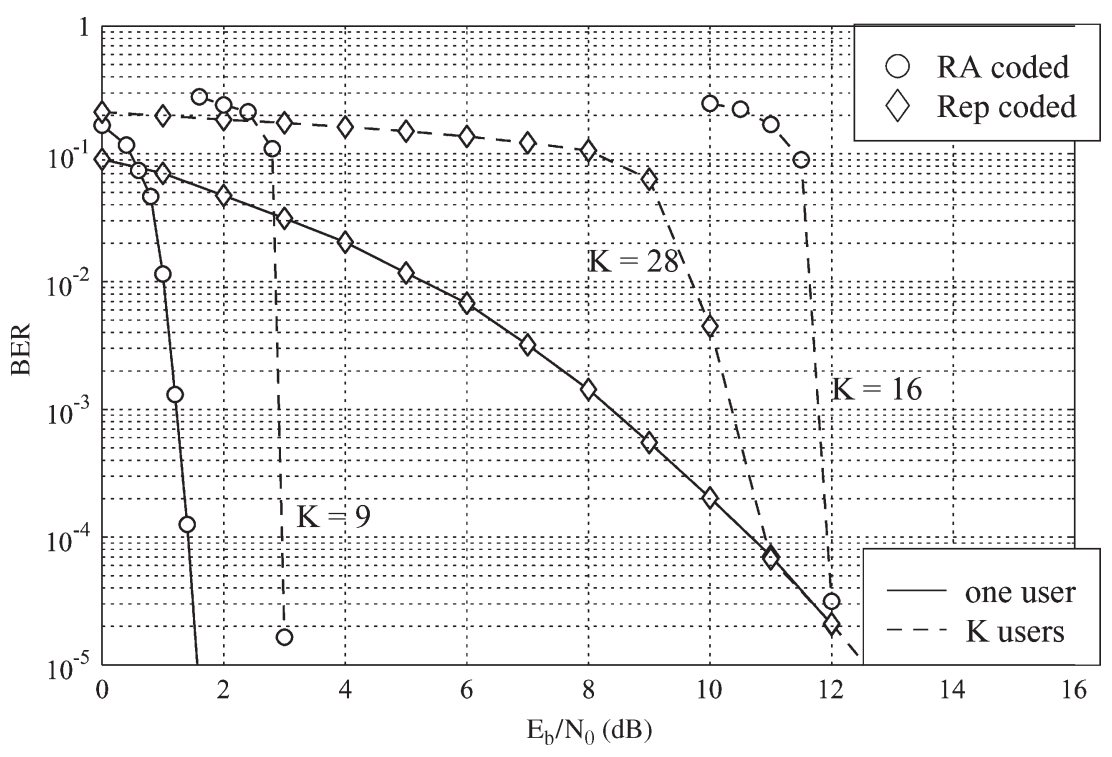

Fig. 5. Performance of the RA and repetition-coded 128-subcarrier MC-IDMA system in a five-path chip-spaced negative exponentially decaying uncorrelated Rayleigh fading channel.

our EXIT chart analysis. The corresponding number of PIC iterations was fixed to $\mathcal{I}_{\mathrm{PIC}}=30$, and the number of iterations within the code was $\mathcal{I}_{\mathrm{RA}}=20$.

\section{TRADEOFFS IN MC-IDMA SYSTEM}

\section{A. Multiplexing Versus Diversity Tradeoffs}

In an MC-IDMA system, at any chip instant, each user's chip may be differentiated by their different FDCTFs, which is essentially equivalent to the concept of spatial-division multiple access (SDMA) [29]. ${ }^{3}$ By contrast, in an MC-IDM system, each stream's signal is differentiated by its unique stream-specific chip interleaver, noting that there is no way of differentiating among the streams at any chip instant since their FDCTFs are identical.

In Fig. 6, we compare the three typical channelcoded/spread-system configurations summarized in Table I, all of which have a total bandwidth expansion factor of $\Omega=8$ and operate at $E_{b} / N_{0}=14 \mathrm{~dB}$. Explicitly, we have the following: 1) Scenario 1, i.e., AWGN channel representing a constant equal-power system; 2) Scenario 2, i.e., MC-IDM representing a multiplexing system designed for supporting the highest possible throughput for a single user, which can also be viewed as a DL broadcast system scenario or the scenario of colocated users/antennas; 3) Scenario 3, i.e., MC-IDMA representing a UL multiple-access system supporting geographically dispersed users/antennas. Based on Fig. 6, we infer the following conclusions.

1) In the noise-limited right-hand-side area of Fig. 6, the interference was almost completely cancelled, resulting in a near-single-user performance. Observe in Fig. 6 that, as expected, Rayleigh fading imposes a detrimental effect on both MC-IDMA- and MC-IDM-type systems

${ }^{3}$ SDMA [29] is a multiple-access technique where each user is differentiated by their user-specific channel impulse response.

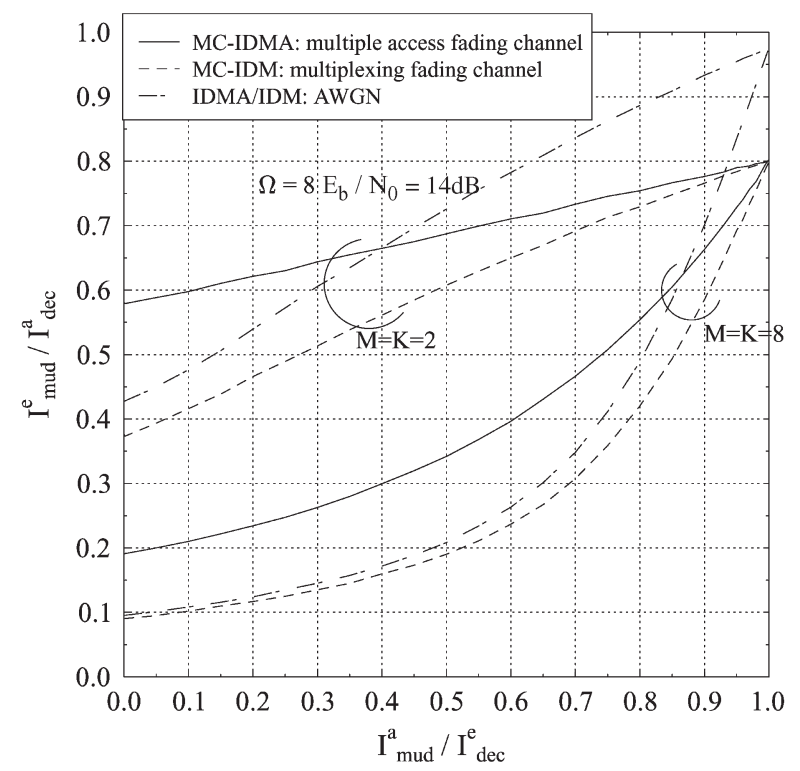

Fig. 6. MUD's EXIT curve for both the 128-subcarrier MC-IDMA and MC-IDM systems for transmission over the five-path uncorrelated Rayleigh fading as well as for an AWGN channel environment.

compared to the AWGN channel when operating at $E_{b} / N_{0}=14 \mathrm{~dB}$.

2) For the multiplexing Scenario 2 using $M=2$ and $M=8$, the extrinsic information recorded for the MCIDM system under fading channel conditions was upper bounded by the AWGN channel's curve. This implies that for the MC-IDM Scenario 2, where the same CIR is experienced by all streams of a given user, the fading always exhibits detrimental effects, leading to either a higher BER or requiring a higher $E_{b} / N_{0}$ value. Nonetheless, the increased single-user throughput may be considered to be the explicit benefit of the system's multiplexing gain.

3) In contrast to the MC-IDM Scenario 2, when the MCIDMA Scenario 3 was employed, in the interferencelimited region of Fig. 6, the system attained an increased 
TABLE I

Summary of the Parameters and Main Results. $\left(E_{b} / N_{0}\right)_{t}$ Represents the Minimum $E_{b} / N_{0}$ Value (IN Decibels) at Which the Channel Code Operates, and $\eta^{*}$ Denotes the Maximum Throughput Expressed in Bits Per Chip

\begin{tabular}{|c|c|c|c|c|c|c|}
\hline \multicolumn{2}{|c|}{ Tradeoff 1: Type of System } & $\Omega$ & $E_{b} / N_{0}$ & Type of Detection & Type of Code & $\eta$ \\
\hline Scenario 1 & SC-IDM / IDMA (AWGN) & 8 & 14 & PIC & 1/8-Rep & 2 \\
\hline Scenario 2 & MC-IDM (Rayleigh) & 8 & 14 & PIC & 1/8-Rep & 2 \\
\hline Scenario 3 & MC-IDMA (Rayleigh) & 8 & 14 & PIC & 1/8-Rep & 3.5 \\
\hline \multicolumn{2}{|c|}{ Tradeoff 2: Type of Code } & $\Omega$ & $\left(E_{b} / N_{0}\right)_{t}$ & Type of System & Type of Detection & $\eta^{*}$ \\
\hline Scheme 1 & 1/64-Rep & 64 & 12 & MC-IDMA / MC-IDM & PIC & $3.625 / 2$ \\
\hline Scheme 2 & 1/2-Cov + 1/32-Rep & 64 & 6 & MC-IDMA / MC-IDM & PIC & $2.75 / 1.375$ \\
\hline Scheme 3 & 1/64-RA & 64 & 3 & MC-IDMA / MC-IDM & PIC & $1.5 / 0.625$ \\
\hline Scheme 4 & 1/4-RA + 1/16-Rep & 64 & 3 & MC-IDMA / MC-IDM & PIC & $2 / 0.75$ \\
\hline \multicolumn{2}{|c|}{ Tradeoff 3: Type of Detection } & $\Omega$ & $E_{b} / N_{0}$ & Type of System & Type of Code & $\eta$ \\
\hline Detection 1 & PIC & 4 & 10 & MC-IDMA & 1/2-Rep +1/2-Conv & 3 \\
\hline Detection 2 & ML & 4 & 10 & MC-IDMA & 1/2-Rep +1/2-Conv & 3 \\
\hline
\end{tabular}

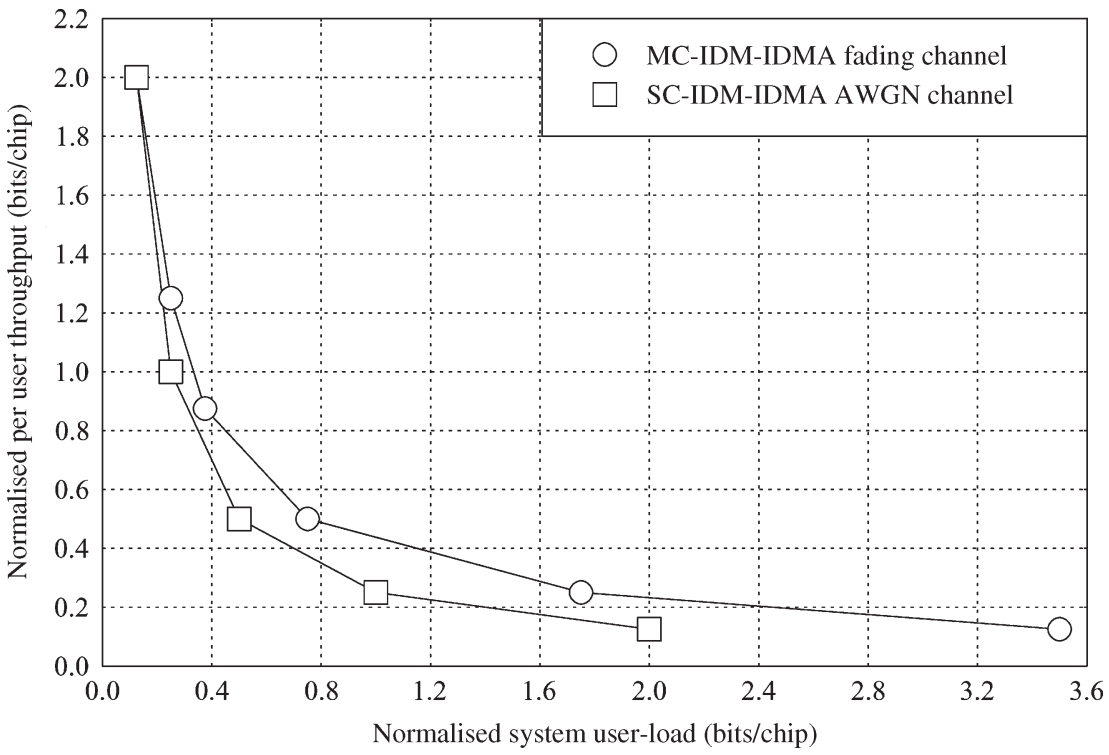

Fig. 7. Tradeoff between the normalized per-user throughput (multiplexing gain) for the 128-subcarrier MC-IDM system and the normalized user load (diversity gain) of 128-subcarrier MC-IDMA in a five-path chip-spaced negative exponentially decaying uncorrelated Rayleigh fading channel.

output extrinsic information under fading channel conditions in comparison with the AWGN channel scenario, where each user was assumed to experience independent fading. This is because each chip can be differentiated by its different FDCTF, resulting in a more reliable estimation of each chip or, equivalently, in a higher extrinsic information. This beneficial effect may be referred to as multiuser diversity [32].

4) Since the a priori information was increased during the consecutive PIC MUD iterations, the interference was essentially cancelled, and as a result, a gradually reduced multiuser diversity benefit was observed. Thus, the improvements recorded as a benefit of multiuser diversity in the MC-IDMA Scenario 3 become less and less compared to the equivalent MC-IDM Scenario 2, as shown in Fig. 6.

Let us now investigate our proposed MC-IDM-IDMA system's multiplexing versus multiuser diversity gain. Fig. 7 shows the associated tradeoffs between the multiplexing gain (per-user throughput) provided by the MC-IDM Scenario 2 and the diversity gain (user-load) supported by the MC-IDMA
Scenario 3. The achievable gains were normalized to the total bandwidth expansion factor of $\Omega=8$, dedicated to length- 8 repetition coding at $E_{b} / N_{0}=14 \mathrm{~dB}$, when communicating over a dispersive Rayleigh fading channel as well as in AWGN channel conditions. It is worth emphasizing that in the MCIDM-IDMA system, it is the total system's normalized user load that is higher than that of the AWGN channel scenario, whereas the individual users' effective throughput is typically low. The maximum normalized per-user throughput provided by the MC-IDM Scenario 2 is shown to be 2 bits/chip in Fig. 7 , whereas the maximum normalized user-load provided by the MC-IDMA Scenario 3 is 3.5 bits/chip in Fig. 7. Our proposed system is thus flexible in terms of assigning the total system throughput to a single IDM user or providing access for several users.

\section{B. Coding Versus Spreading Tradeoffs}

1) Joint Coding and Spreading Design: Let us now continue our investigations by fixing the bandwidth expansion factor to $\Omega=64$ and find the maximum number of users supported by different channel-coding combinations at various 


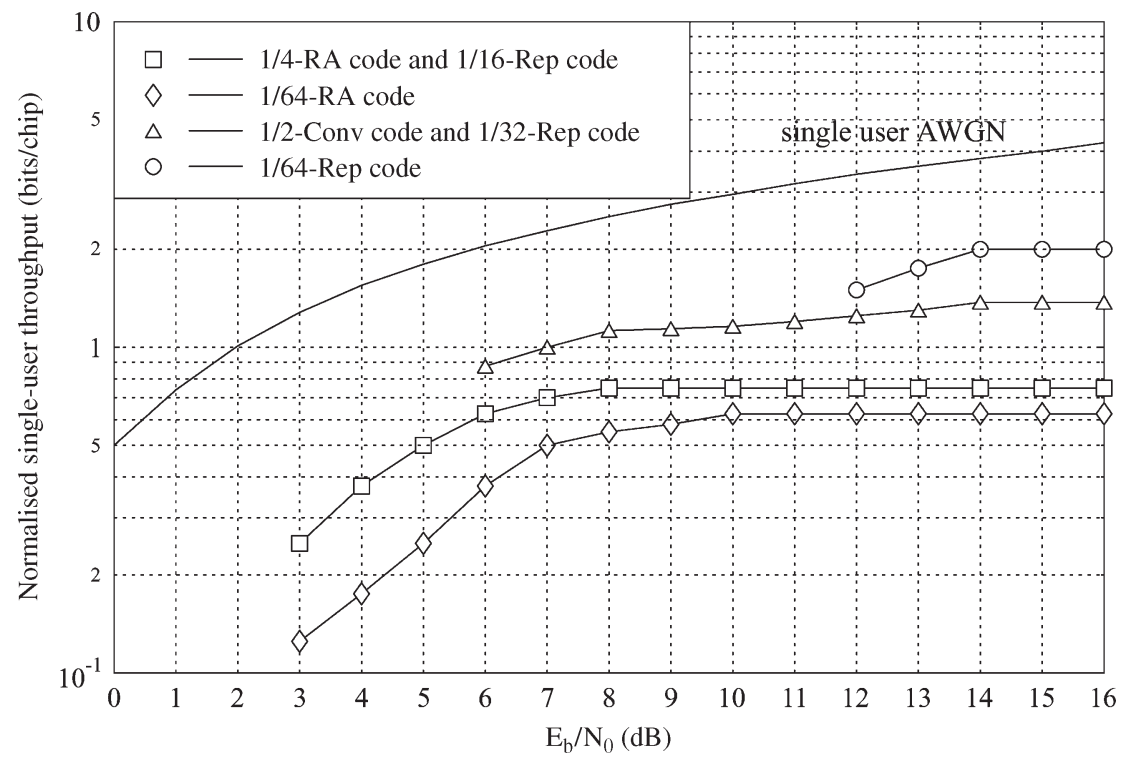

Fig. 8. Normalized per-user throughput versus $E_{b} / N_{0}$ performance of the 128 -subcarrier MC-IDM for a fixed bandwidth expansion factor of $\Omega=64$ and for $P_{e}=10^{-5}$ using the various channel-coding schemes considered in a five-path chip-spaced negative exponentially decaying uncorrelated Rayleigh fading channel.

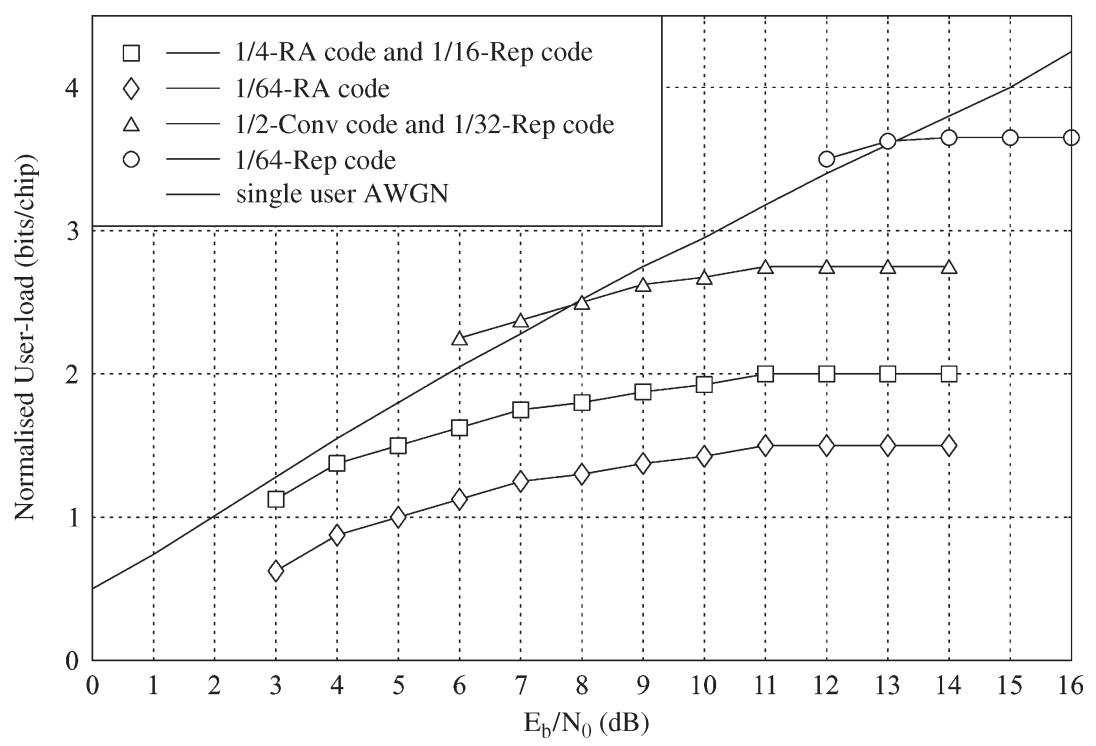

Fig. 9. Normalized user load versus $E_{b} / N_{0}$ performance of the 128-subcarrier MC-IDMA for a fixed-bandwidth expansion factor of $\Omega=64$ and for $P_{e}=10^{-5}$ using the various channel-coding schemes considered in a five-path chip-spaced negative exponentially decaying uncorrelated Rayleigh fading channel.

$E_{b} / N_{0}$ values while maintaining a given target BER of $10^{-5}$ with the aid of EXIT charts. ${ }^{4}$ Four typical channel-coding combinations are investigated, as shown in Table I, namely,

\footnotetext{
${ }^{4}$ The EXIT chart can be used to obtain an estimate on the bit error probability after an arbitrary number of iterations. The soft bit values outputted by the MUD after a given number of iterations can be expressed using the $a$ posteriori LLRs, which is the sum of the a priori LLRs and the extrinsic LLRs denoted by $L_{\mathrm{mud}, k}=L_{\text {mud }, k}^{a}+L_{\text {mud }, k}^{e}$. It is Gaussian distributed since both $L_{\text {mud }, k}^{a}$ and $L_{\text {mud }, k}^{e}$ are Gaussian. The coded bit error probability is $P e \approx Q(\sigma / 2)$, where $\sigma$ is the variance of $L_{\mathrm{mud}, k}$, and $Q$ is the well-known Gaussian $Q$-function. Since $L_{\text {mud }, k}^{a}$ and $L_{\text {mud }, k}^{e}$ are independent, then $\sigma^{2}=\sigma_{a}^{2}+\sigma_{e}^{2}$, where $\sigma_{a}$ and $\sigma_{e}$ represent the variance of the a priori LLRs $L_{\text {mud }, k}^{a}$ and the extrinsic LLRs $L_{\text {mud, }}^{e}$, respectively. The values of $\sigma_{a}$ and $\sigma_{e}$ can be obtained from the corresponding mutual information $I_{\mathrm{mud}, k}^{a}$ and $I_{\mathrm{mud}, k}^{e}$, since the mutual information is a monotonically increasing function of the LLRs.
}

1) Scheme 1, i.e., rate-1/64 repetition code; 2) Scheme 2, i.e., the concatenation of a rate- $1 / 2$ convolutional code and rate1/32 repetition code; 3) Scheme 3, i.e., rate-1/64 RA code; and 4) Scheme 4 , i.e., the concatenation of a rate-1/4 RA code and rate-1/16 repetition code. In Figs. 8 and 9, the rate-1/64 pure repetition-coded MC-IDM and MC-IDMA Scheme 1 shows the highest multiple-access capability at high $E_{b} / N_{0}$ values for all the codes considered. In a channel-coded system scenario, as expected, the convolutional-coded Scheme 2 supports a higher number of users than the RA-coded Scheme 3 and Scheme 4 for SNR values in excess of $E_{b} / N_{0}=5 \mathrm{~dB}$, since it has a higher multiple-access capability, as shown in Fig. 3. However, the RA-coded Schemes 3 and 4 are capable of reliably operating at lower $E_{b} / N_{0}$ values as a benefit of their higher error correction capability. 
RA-coded Schemes 3 and 4 were also compared in Figs. 8 and 9, where the number of RA-decoding iterations was fixed to $\mathcal{I}_{\mathrm{RA}}=20$. The low-rate RA-coded Scheme 3 using an insufficient number of decoding iterations has roughly the same error correction capability but an inferior multiple-access capability in comparison with the RA-coded Scheme 4 represented by the square-shaped legends. The number of iterations required by the rate-1/64 RA-coded Scheme 3 was found to be $\mathcal{I}_{\mathrm{RA}}=80$ to have superior performance in comparison to Scheme 4.

Importantly, the achievable normalized user load of both the convolutional-coded MC-IDMA Scheme 2 and the repetitioncoded MC-IDMA Scheme 1 communicating over fading channels is quite remarkable in comparison with the single-user AWGN scenario, as shown in Fig. 9. This is a benefit of the previously discussed multiuser diversity. In fact, the achievable user-load improvements increase without limits upon increasing $E_{b} / N_{0}$, as stated in [32] in the context of DS-CDMA. These benefits can further be increased by using MUDs having a near-single-user performance or optimized unequal power/rate allocation [4].

2) Comparisons and Conclusion: Based on the above analysis, we found that the joint coding and spreading design of the MC-IDM and MC-IDMA systems has to take into account the specific $E_{b} / N_{0}$ values encountered. At high $E_{b} / N_{0}$ values, DS spreading dispensing with FEC was capable of supporting the highest normalized throughput. By contrast, at low $E_{b} / N_{0}$ values, the employment of explicit channel coding becomes crucial since it improves the achievable BER at the cost of reducing the attainable multiple-access capability.

Yue and Wang [24] found that the employment of channel coding was always beneficial for MF-based iterative MUDs in DS-CDMA systems, regardless of the $E_{b} / N_{0}$ values encountered. When considering the MC-IDM and MC-IDMA systems, which also employ MF-based iterative MUD, our conclusions stated above were similar to those drawn for a minimum mean square error (MMSE)-based iterative MUD in a DS-CDMA system, as reported in [24], which potentially requires highcomplexity matrix inversion operations. This suggests that the employment of classic CDMA DS spreading codes should be replaced by repetition codes in the context of both MC-IDM and MC-IDMA in the interest of benefiting from both its lowcomplexity MF-based detection and an improved bandwidth efficiency.

In classic DS-CDMA, the different MUDs exploit the diverse correlation properties of DS spreading codes. Hence, they result in different channel-coding versus spreading tradeoffs, depending on the specific features of the MUDs employed. The MUD of MC-IDM and MC-IDMA operates on a chip-by-chip basis; thus, the joint coding and spreading design becomes a singlejoint low-rate code design problem. A rule of thumb when designing MC-IDM and IDMA systems for operating in various channel conditions at a given $E_{b} / N_{0}$ constraint and at an affordable decoding complexity is that of opting for the specific FEC channel code having the highest rate, which is "just" capable of meeting the specific target BER requirements, and then dedicating the rest of the affordable bandwidth expansion to DS spreading, i.e., to DS repetition codes.

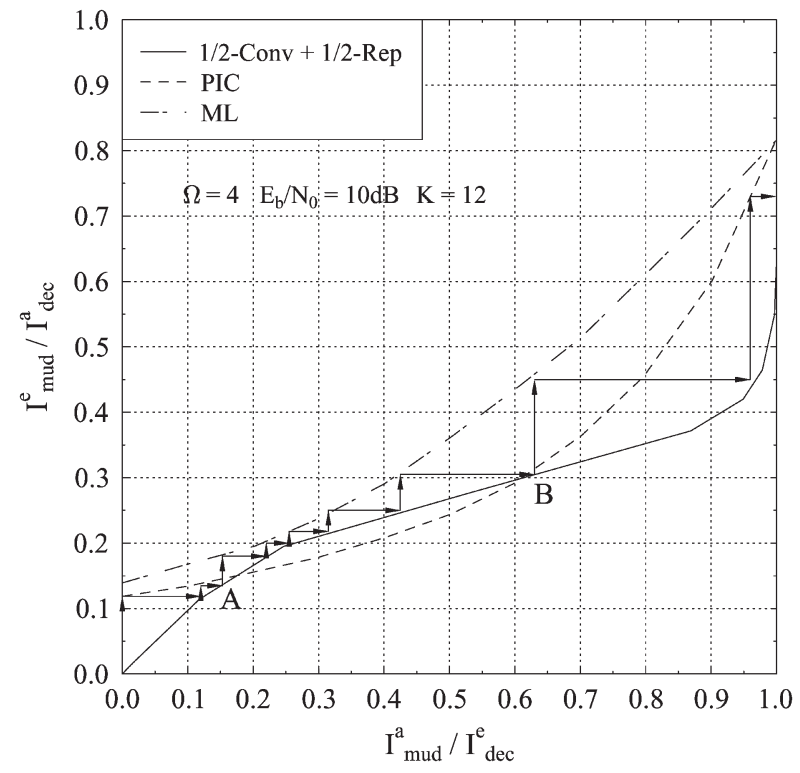

Fig. 10. EXIT chart analysis of different MUDs.

\section{Complexity Versus Performance Tradeoffs}

For the sake of achieving higher user load, more powerful MUD algorithms are necessary instead of employing sophisticated power allocation schemes. In this section, we investigate the EXIT curves of the optimum ML MUD and the currently proposed PIC MUD. Furthermore, a hybrid MUD is proposed, which is capable of approaching the ML MUD's performance at a reduced complexity. Fig. 10 compares the $T_{\text {mud }}$ function of the $K=12$ user MC-IDMA system communicating over a dispersive Rayleigh fading channel scenario having a bandwidth expansion factor of $\Omega=4$ and $E_{b} / N_{0}=10 \mathrm{~dB}$ when employing a suboptimum MF-based PIC MUD and ML MUD. At the left of Fig. 10, which corresponds to the interferencelimited region, the ML MUD only outputs marginally higher extrinsic information than the PIC MUD. This implies that the achievable multiuser diversity is sufficiently high for almost entirely compensating for the suboptimal nature of the MF-based PIC MUD algorithm compared to the optimum ML MUD. As the amount of available a priori information increases during the consecutive iterations, the gap between these two $T_{\text {mud }}$ curves becomes more substantial owing to the reduced multiuser diversity and the suboptimal nature of the MF-based PIC MUD. Ultimately, these two $T_{\text {mud }}$ curves tend to the corresponding single-user performance when the effects of MUI have been more or less eliminated.

However, the complexity order of an ML detector exponentially increases with the number of users according to $\mathcal{O}\left(2^{K}\right)$, whereas that of a PIC detector linearly increases with the number of users obeying $\mathcal{O}(K)$ according to [3]. Hence, designing a MUD scheme that is capable of approaching the ML performance with reduced complexity is of interest. This may be achieved with the aid of a hybrid MUD [33]. As shown in Fig. 10, during the initial few iterations, we activate the PIC MUD. Then, the ML MUD is activated to overcome the PIC MUD's EXIT curve intersection between points A and B in Fig. 10, where the PIC MUD's EXIT curve $T_{\text {mud }}$ is below 
the EXIT curve $T_{\mathrm{dec}}$ of DEC. After this bottleneck, the PIC MUD is reactivated in the interest of reducing the complexity imposed. Additionally, other near-ML MUDs can be employed for the sake of further reducing the complexity imposed, such as genetic-algorithm-aided and sphere-decoding-aided MUDs [29].

\section{CONCLUSION}

In this paper, we have proposed the novel generalized model of MC-IDM-IDMA and analyzed its convergence behavior with the aid of EXIT charts. The differences between MC-IDM and MC-IDMA were investigated, and the multiuser diversity benefits of MC-IDMA were revealed. The proposed system is flexible in terms of either assigning the total system throughput to a single user or providing a multiple-access capability for several users. The associated coding versus DS spreading tradeoffs were discussed, and our findings were compared with the characteristics of traditional DS-CDMA. It was found that in contrast with the separate DS spreading and channel-coding design of traditional DS-CDMA systems, the DS spreading operation should be replaced by a repetition code in the context of IDMA to be jointly designed with the FEC code to improve the achievable bandwidth efficiency. A general design rule was also provided for the low-rate codes of our systems. Furthermore, the complexity versus performance tradeoffs were discussed by investigating two MUD EXIT curves, and a reduced-complexity hybrid MUD concept was provided. Our future research will consider the joint design of coding and spreading for high-throughput multilevel modulation.

\section{ACKNOWLEDGMENT}

The authors would like to thank the anonymous reviewers for their valuable suggestions and $\mathrm{L}$. Xu for his suggestion of the hybrid MUD concept. Fully detailed technical reports on this research are available to Industrial Members of Mobile VCE.

\section{REFERENCES}

[1] P. Frenger, P. Orten, and T. Ottosson, "Code-spread CDMA using maximum free distance low-rate convolutional codes," IEEE Trans. Wireless Commun., vol. 48, no. 1, pp. 135-144, Jan. 2000.

[2] R. H. Mahadevappa and J. G. Proakis, "Mitigating multiple access interference and intersymbol interference in uncoded CDMA systems with chip-level interleaving," IEEE Trans. Wireless Commun., vol. 1, no. 4, pp. 781-792, Oct. 2002.

[3] L. Ping, L. Liu, K. Wu, and L. W. K. , "Interleave division multipleaccess," IEEE Trans. Wireless Commun., vol. 5, no. 4, pp. 938-947, Apr. 2006.

[4] L. Liu, J. Tong, and L. Ping, "Analysis and optimization of CDMA systems with chip-level interleavers," IEEE J. Sel. Areas Commun., vol. 24, no. 1, pp. 141-150, Jan. 2006.

[5] H. Schoeneich and P. A. Hoeher, "Adaptive interleave-division multiple access-A potential air interference for $4 \mathrm{G}$ bearer services and wireless LANs," in Proc. WOCN, Muscat, Oman, Jun. 7-9, 2004, pp. 179-182.

[6] P. A. Hoeher and H. Schoeneich, "Interleave-division multiple access from a multiuser point of view," in Proc. 5th Int. Symp. Turbo Codes Related Topics Connection 6th Int. ITG-Conf. Source Channel Coding, Munich, Germany, Apr. 3-7, 2006, pp. 140-144.

[7] L. Hanzo, L.-L. Yang, E.-L. Kuan, and K. Yen, Single- and Multi-Carrier DS-CDMA: Multi-User Detection, Space-Time Spreading, Synchronisation, Networking and Standards. New York: Wiley-IEEE Press, 2003.
[8] L. Ping, W. K. Leung, and K. Y. Wu, "Low-rate turbo-Hadamard codes," IEEE Trans. Inf. Theory, vol. 49, no. 12, pp. 3213-3224, Dec. 2003.

[9] L. Ping, X. Huang, and N. Phamdo, "Zigzag codes and concatenated zigzag codes," IEEE Trans. Inf. Theory, vol. 47, no. 2, pp. 800-807, Feb. 2001.

[10] P. A. Hoeher and H. Schoeneich, "Semi-blind pilot-layer aided channel estimation with emphasis on interleave-division multiple access systems," in Proc. IEEE GLOBECOM, St. Louis, MO, Nov./Dec. 2005, pp. 3513-3517.

[11] K. Kusume and G. Bauch, "CDMA and IDMA: Iterative multiuser detections for near-far asynchronous communications," in Proc. IEEE 16th Int. Symp. Pers., Indoor Mobile Radio Commun., Berlin, Germany, Sep. 11-14, 2005, pp. 426-431.

[12] K. Li, X. Wang, G. Yue, and L. Ping, "A low-rate code-spread and chipinterleaved time-hopping UWB system," IEEE J. Sel. Areas Commun., vol. 24, no. 4, pp. 864-870, Apr. 2006.

[13] R. Zhang and L. Hanzo, "EXIT chart based joint code-rate and spreading-factor optimisation of single-carrier interleave division multiple access," in Proc. IEEE WCNC, Hong Kong, Mar. 11-15, 2007, pp. 735-739.

[14] S. Zhou, Y. Li, M. Zhao, X. Xu, J. Wang, and Y. Yao, "Novel techniques to improve downlink multiple access capacity for beyond 3G," IEEE Commun. Mag., vol. 43, no. 1, pp. 61-69, Jan. 2005.

[15] K. Kusume and G. Bauch, "Some aspects of interleave division multiple access in ad hoc networks," in Proc. 5th Int. Symp. Turbo Codes Related Topics Connection 6th Int. ITG-Conf. Source Channel Coding, Munich, Germany, Apr. 3-7, 2006, pp. 135-139.

[16] P. Wang, J. Xiao, and L. Ping, "Comparison of orthogonal and nonorthogonal approaches to future wireless cellular systems," IEEE Veh. Technol. Mag., vol. 1, no. 3, pp. 4-11, Sep. 2006.

[17] I. Mahafeno, C. Langlais, and C. Jego, "OFDM-IDMA versus IDMA with ISI cancellation for quasi-static Rayleigh fading multipath channels," in Proc. 4th Int. Symp. Turbo Codes Related Topics Connection 6th Int. ITG-Conf. Source Channel Coding, Munich, Germany, Sep. 3-7, 2006, pp. $140-144$.

[18] R. Zhang and L. Hanzo, "Iteratively detected multicarrier interleave division multiple access," in Proc. IEEE MICROCOLL, Budapest, Hungary, May 14-16, 2007, pp. 381-385. [Online]. Available: http://eprints.ecs.soton.ac.uk/13914/

[19] L. Hanzo, J. Blogh, and S. Ni, 3G, HSDPA, HSUPA and Intelligent FDD Versus TDD Networking: Smart Antennas and Adaptive Modulation. New York: Wiley-IEEE Press, 2007.

[20] M. C. Reed, C. B. Schlegeland, P. D. Alexander, and J. A. Asenstorfer, "Iterative multiuser detection for CDMA with FEC: Near-single-user performance," IEEE Trans. Commun., vol. 46, no. 12, pp. 1693-1699, Dec. 1998.

[21] X. Wang and H. V. Poor, "Iterative (turbo) soft interference cancellation and decoding for coded CDMA," IEEE Trans. Commun., vol. 47, no. 7, pp. 1046-1061, Jul. 1999.

[22] S. ten Brink, "Convergence behavior of iteratively decoded parallel concatenated codes," IEEE Trans. Commun., vol. 49, no. 10, pp. 1727-1737, Oct. 2001.

[23] K. Li and X. Wang, "EXIT chart analysis of turbo multiuser detection," IEEE Trans. Wireless Commun., vol. 4, no. 1, pp. 300-311, Jan. 2005.

[24] G. Yue and X. Wang, "Coding-spreading tradeoff in LDPC-coded CDMA with turbo multiuser detection," IEEE Trans. Wireless Commun., vol. 3 , no. 5, pp. 1734-1745, Sep. 2004.

[25] H. Wei and L. Hanzo, "Coding against spreading gain optimisation of nonbinary BCH coded CDMA system," Electron. Lett., vol. 41, no. 14 pp. 816-817, Jul. 2005.

[26] S. Verdu and S. Shamai, "Spectral efficiency of CDMA with random spreading," IEEE Trans. Inf. Theory, vol. 45, no. 2, pp. 622-640, Mar. 1999.

[27] D. Tse and P. Viswanath, Fundamentals of Wireless Communication. Cambridge, U.K.: Cambridge Univ. Press, 2005.

[28] L. Hanzo, T. Liew, and B. Yeap, Turbo Coding, Turbo Equalisation and Space-Time Coding for Transmission Over Fading Channels. New York: Wiley-IEEE Press, 2002.

[29] L. Hanzo and T. Keller, OFDM and MC-CDMA: A Primer. New York: Wiley-IEEE Press, 2006.

[30] J. Boutros and G. Caire, "Iterative multiuser joint decoding: Unified framework and asymptotic performance analysis," IEEE Trans. Inf. Theory, vol. 48, no. 7, pp. 1772-1793, Jul. 2002.

[31] J. Jin, A. Khandekar, and R. J. McEliece, "Irregular repeat-accumulate codes," in Proc. 2nd Int. Conf. Turbo Codes, Munich, Germany, Sep. 4-7, 2000, pp. 125-127. 
[32] S. Shamai and S. Verdu, "The impact of frequency-flat fading on the spectral efficiency of CDMA," IEEE Trans. Inf. Theory, vol. 47, no. 4, pp. 1302-1327, May 2001.

[33] L. Xu, R. Zhang, S. Chen, and L. Hanzo, "EXIT-chart aided hybrid multiuser detector design for frequency-domain-spread chip-interleaved MC-CDMA," in Proc. IEEE VTC-Spring, 2008, pp. 1816-1820.

Rong Zhang received the B.E. degree in information engineering from Southeast University, Nanjing, China, in 2003 and the M.Sc. degree with distinction in radio frequency communication systems from the University of Southampton, Southampton, U.K., in 2005 . He is currently working toward the $\mathrm{Ph} . D$. degree with the Communications Research Group, School of Electronics and Computer Science, University of Southampton.

His research interests include channel coding, OFDM, CDMA/IDMA, and colocated and distributed MIMO systems.

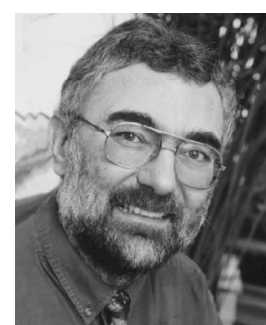

Lajos Hanzo (M'91-SM'92-F'04) received the Dipl.Ing. degree in electronics and the Ph.D. degree from the Technical University of Budapest, Budapest, Hungary, in 1976 and 1983, respectively, and the D.Sc. degree from the University of Southampton, Southampton, U.K., in 2004.

During his career in telecommunications, he has held various research and academic posts in Hungary, Germany, and the U.K. Since 1986, he has been with the Department of Electronics and Computer Science, University of Southampton, where he holds the Chair in telecommunications. He is an enthusiastic liaison between industry and academia, and he offers a range of industrial courses. He has coauthored 15 Wiley and IEEE press books totaling 10000 pages on mobile radio communications, has published about 700 research papers, has organized and chaired conferences, has presented various keynote and overview lectures, and has been awarded a number of distinctions. He currently heads an academic research team working on a range of research projects in the field of wireless multimedia communications sponsored by the industry, the Engineering and Physical Sciences Research Council U.K., the European IST Program, and the Mobile Virtual Centre of Excellence, U.K.

Dr. Hanzo is a Fellow of the Royal Academy of Engineering and the Institution of Electrical Engineers. He is also an IEEE Distinguished Lecturer of both the Communications as well as the Vehicular Technology (VT) Societies. $\mathrm{He}$ is an Editorial Board Member of the PROCEEDINGs OF THE IEEE and a Governor of the IEEE VT Society. 